\title{
PROJECTIVE MAXIMAL RIGHT IDEALS OF SELF-INJECTIVE RINGS
}

\author{
O. A. S. KARAMZADEH
}

ABSTRACT。 It is proved that a projective maximal right ideal $M$ of a self-injective ring $R$ is of the form $M=e R+J(R)$. It is also shown that if every maximal right ideal of a self-injective ring $R$ is projective, then $R$ must be Artin semisimple.

A ring $R$ is called self-injective if $R$ is injective as a right $R$-module. By a regular ring we mean regular in the sense of Von Neumann [4].

Let $M$ be a right $R$-module; a submodule $P$ of $M$ is said to be essential if $P \cap N \neq(0)$, for any submodule $0 \neq N$ of $M$; we denote this by $M$ ' $\supset$. Then the singular submodule $Z(M)$ is defined by $Z(M)=\{x \in M \mid R$ ' $\supset$ Ann $(x)\}$.

It is easy to see that if $R$ is a regular ring, then $Z(R)=0$.

It is also well known that if $R$ is a self-injective ring with the Jacobson radical $J=J(R)$, then $J=Z(R)$ and $R / J$ is a self-injective regular ring, and finitely many orthogonal idempotents of $R / J$ can be lifted to orthogonal idempotents of $R$ (see [2]).

Throughout this paper, $R$ will denote an associative ring which does have a unity. The reader is referred to [2] for basic results on semiperfect rings.

Proposition 1. Let $R$ be a self-injective regular ring; then a maximal right ideal which is projective is a direct summand.

Proof. Let $M$ be a maximal right ideal which is projective as a right $R$-module; then $M=\Sigma_{i \in I} \bigoplus e_{i} R ; e_{i}^{2}=e_{i} \forall i \in I$ (see [1]). Since every maximal right ideal is either essential or a direct summand, we must assume that $M$ is essential and, therefore, $I$ is infinite.

Without loss of generality we can assume $e_{i} e_{j}=0, i \neq j$; for if $p_{i}: M \rightarrow$ $e_{i} R$ and $k_{i}: e_{i} R \rightarrow M$ are the natural projection and injection, respectively,

Received by the editors November 20, 1973 and, in revised form, February 25, 1974.

AMS (MOS) subject classifications (1970). Primary 16A30, 16A52.

Key words and phrases. Self-injective ring, regular ring, essential submodule, singular submodule, semiperfect ring. 
and $\alpha_{i}=k_{i} \rho_{i}$, then $\alpha_{i} \in \operatorname{Hom}_{R}(M, M)$ and $\alpha_{i}^{2}=\alpha_{i} \forall i \in I$ and $\alpha_{i} \alpha_{j}=0$, $i \neq j$. Clearly $a=\Sigma \alpha_{i} a \forall a \in M$, where $\alpha_{i} a=0$ for almost all $i \in I$. So $M=\Sigma \bigoplus \alpha_{i} M$ and, as $R$ is injective, there exists for each $\alpha_{i}$ in $\operatorname{Hom}_{R}(M, M)$ an element $e_{i}^{\prime} \in R$ such that $\alpha_{i} m=e_{i}^{\prime} m \forall m \in M$. Then since $Z(R)=0$, we get $e_{i}^{\prime}=e_{i}^{\prime 2} \forall i \in I$ and $e_{i}^{\prime} e_{j}^{\prime}=0, i \neq j$.

Now we assume $M=\Sigma_{i \in I} \bigoplus e_{i} R, e_{i}^{2}=e_{i}, e_{i} e_{j}=0, i \neq j$. Since $I$ is infinite we can have $I=I_{1} \cup I_{2}$, where $I_{1}$ and $I_{2}$ are both infinite subsets of 1 . Set $M_{1}=\Sigma_{i \in I_{1}} \bigoplus e_{i} R, M_{2}=\Sigma_{j \in I_{2}} \bigoplus e_{j} R$.

Now if $p_{i}: M \rightarrow M_{i}, i=1,2$, is the natural projection, there exists $a_{i}$, $i=1,2$, in $R$ such that $p_{i}(m)=a_{i} m, i=1,2, \forall m \in M$. Clearly $a_{1} a_{2} M=$ $a_{2} a_{1} M=0$, so $a_{1} a_{2}=a_{2} a_{1}=0$. We claim that either $a_{1}$ or $a_{2}$ belongs to $M$, for if $a_{2} \notin M$, then $a_{2} R+M=R$ implies $1=a_{2} r+m, r \in R, m \in M$; then multiply.ing by $a_{1}$ we get $a_{1}=a_{1} a_{2} r+a_{1} m \in M$. So a ssume $a_{1} \in M$, then $a_{1}=m_{1}+m_{2}, m_{i} \in M_{i}, i=1,2$. Multiply.ing by an element $x \in M_{1}$ we get $a_{1} x=m_{1} x+m_{2} x$ which implies $x=m_{1} x, m_{2} x=0$; in particular, $m_{1}=m_{1}^{2}$. This shows that $M_{1}=m_{1} R$ and so $m_{1} e_{i}=e_{i} \forall i \in I_{1}$.

If $m_{1}=\Sigma_{i \in A} e_{i} r_{i}$, where $A$ is a finite subset of $I_{1}$, then $e_{i} m_{1}=0$ $\forall i \in I_{1}-A$, and also $e_{i}=e_{i}^{2}=\left(m_{1} e_{i}\right)^{2}=0 \forall i \in I_{1}-A$, a contradiction. Hence $I$ must be finite.

The following result investigates the projective maximal right ideals in a self-injective ring.

Corollary 1. If $R$ is a self-injective ring and $M$ a projective maximal right ideal, then $M=e R+J$, where $e=e^{2}$.

Proof. By using the dual basis lemma and the fact that $R$ is injective, it is easy to see that a right ideal $M$ is projective if and only if there exists a collection $\left\{m_{i}\right\} \subset M$ such that for all $m \in M, m_{i} m=0$ for almost all $i$ and $m=\Sigma m_{i} m$; hence if $M$ is a maximal right ideal, $M / J$ is a projective maximal right ideal of $R / J$ and, in view of Proposition 1 and the fact that idempotents lift, we have $M / J=(e R+J) / J, e=e^{2}$ which implies $M=e R+J$.

The following lemma is well known (see [2, p. 67]).

Lemma 1. If every maximal right ideal of a ring $R$ is a direct summand, then $R$ is Artin semisimple.

It is trivial to see that if the Jacobson radical of a ring $R$ is injective it must be zero. We also have the following result:

Lemma 2. If $R$ is a self-injective ring, then no nonzero right ideal in. side the Jacobson radical is projective. 
Proof. Let $0 \neq I$ be a projective right ideal contained in $J$, and let $\left\{a_{i}\right\}$ be a collection of elements of $I$ such that $a_{i} x=0$ for almost all $i$ and $\forall x \in I$ and also $x=\Sigma a_{i} x$. But $\Sigma_{a_{i} x \neq 0} a_{i} \in J$ shows that $1-\Sigma_{a_{i} x \neq 0} a_{i}$ is a unit, so $x\left(1-\Sigma_{a_{i} x \neq 0} a_{i}\right)=0$ implies $x=0$, a contradiction.

Proposition 1, together with Lemma 1, yields the following result.

Corollary 2. There does not exist a non-Artinian self-injective regular ring in which every maximal right ideal is countably generated.

Proof. In a regular ring, since every countably generated right ideal is a direct sum of principal right ideals, it is projective.

Osofsky has shown [3] that a self-injective ring $R$ which is right hereditary must be Artin semisimple. We prove a generalization of this result.

Corollary 3. If every maximal right ideal of a self-injective ring $R$ is projective, then $R$ is Artin semisimple.

Proof. Since every maximal right ideal $M$ is of the form $M=e R+J$, it is sufficient to show that $J=0$. Clearly $R / J$ is Artin semisimple and, as idempotents lift modulo $J, R$ becomes a semiperfect ring. So $R$ contains a finite orthogonal set of primitive idempotents $\left\{e_{1}, e_{2}, \ldots, e_{n}\right\}$ such that $1=e_{1}+e_{2}+\cdots+e_{n}$, and it is well known that for each $i, e_{i} R / e_{i} J$ is a simple $R$-module. Therefore for each $i, e_{i} J \oplus \Sigma_{i \neq k} \oplus e_{k} R$ is a maximal right ideal, so $J=e_{1} J \oplus \ldots \oplus e_{n} J$ is projective, which implies $J=0$.

I wish to thank the referee whose valuable suggestions led me to answer my question in an earlier version of this paper.

\section{REFERENCES}

1. I. Kaplansky, Projective modules, Ann. of Math. (2) 68 (1958), 372-377. MR 20 \#6453.

2. J. Lambek, Lectures on rings and modules, Blaisdell, Waltham, Mass., 1966. MR $34 \# 5857$.

3. B. L. Osofsky, Rings all of whose finitely generated modules are injective, Pacific J. Math. 14 (1964), 645-650. MR 28 \#5090.

4. J. Von Neumann, On regular rings, Proc. Nat. Acad. Sci. U.S.A. 22 (1936), 707-713.

DEPARTMENT OF MATHEMATICS, UNIVERSITY OF EXETER, EXETER, ENGLAND

Current address: Department of Mathematics, Jundi-Shapur University, Ahwaz, Iran 